\title{
HOMOTOPY GROUPS OF COMPACT HAUSDORFF SPACES WITH TRIVIAL SHAPE
}

\author{
JAMES E. FELT
}

\begin{abstract}
Given a collection $\left\{\pi_{n}: n=1,2, \cdots\right\}$ of countable groups such that $\pi_{i}$ is abelian and admits $\pi_{1}$ as a group of operators for $i \geqq 2$, we construct here an arcwise connected compact metric space of trivial shape whose $j$ th homotopy group is isomorphic to $\pi_{j}$ for $j=1,2, \cdots$. The isomorphisms preserve the action of the first group on the higher groups. Thus, the homotopy groups of a compact metric space of trivial shape may be quite arbitrary.
\end{abstract}

Introduction. The notion of shape in the category $C$ of compact Hausdorff spaces and continuous maps and in $C^{\prime}$, the category of compact Hausdorff pairs and continuous maps of pairs has been formulated by Mardesič and Segal [6] and by Holsztyński [2]. The two approaches were shown to be equivalent in [4] and Holsztyński has shown in [2] that the shape functor is continuous with respect to inverse limits in $C$ and inverse limits in $C^{\prime}$. This continuity, together with a result from [3], is used in this paper to show that any arcwise connected compact Hausdorff space $X$ can be embedded in an arcwise connected compact Hausdorff space $Y$ with trivial shape and homotopy groups isomorphic to those of $X$. The isomorphisms are such that the action of the first group on the higher groups is preserved. The theorem of [8] is then used to show that given a collection $\left\{\pi_{n}: n=1,2, \cdots\right\}$ of countable groups with $\pi_{i}$ abelian and admitting $\pi_{1}$ as a group of operators for $i \geqq 2$, there is an arcwise connected compact metric space with trivial shape and $j$ th homotopy group isomorphic to $\pi_{j}$ for $j=1,2, \cdots$. The isomorphisms are such that the action of the first group on the higher groups is preserved. The construction of this space was suggested to the author by Professor James Keesling. The author wishes to thank him for his guidance.

1. Spaces with trivial shape. Notation and definitions are taken from [6]. A directed set $(A, \leqq)$ is said to be closure-finite if each element of $A$ is preceded by only a finite number of elements of $A$.

1.1. Definition. Let $P$ be a compact Hausdorff space. $P$ has trivial shape if, whenever $P$ is the limit of $\left\{P_{\alpha} ; p_{\alpha \beta} ; \alpha \leqq \beta \in A\right\}$, an inverse system

Received by the editors May 15, 1973.

AMS (MOS) subject classifications (1970). Primary 55D99; Secondary 55E05.

Key words and phrases. Shape, homotopy group, shape group.

(c) American Mathematical Society 1974 
of $A N R$ 's in $C$, with $A$ closure-finite, the following is true:

For each $\alpha \in A$ there is a $\beta \in A, \alpha \leqq \beta$, such that $p_{\alpha \beta}$ is homotopic to a constant map.

Suppose $P_{0}$ is a closed subset of $P$. The pair $\left(P, P_{0}\right)$ has trivial shape if whenever $\left(P, P_{0}\right)$ is the limit of $\left\{\left(P_{\alpha}, P_{0 \alpha}\right) ; p_{\alpha \beta} ; \alpha \leqq \beta \in A\right\}$, an inverse system of $A N R$ pairs in $C^{\prime}$, with $A$ closure-finite, $(*)$ holds with $p_{\alpha \beta}$ and a constant map homotopic as maps of pairs. In [6] it is shown that if $(*)$ holds for one such inverse system with limit $P\left(\left(P, P_{0}\right)\right)$, then $(*)$ holds for any such system with limit $P\left(\left(P, P_{0}\right)\right)$.

1.2. Lemma (Hyman [3]). Every compact metric space having trivial shape is the inverse limit of a sequence of compact metric AR's.

1.3. Lemma. Every compact Hausdorff space with trivial shape is the inverse limit of compact metric spaces each having trivial shape. (The analogous statements for pairs and pointed spaces are also true.)

Proof. Let $P$ be a compact Hausdorff space with trivial shape. By Theorem 7 on p. 45 of [6], there is a presentation $\left\{P ; p_{\alpha} ; \alpha \in A\right\}$ of $P$ as the limit of an inverse system $\left\{P_{\alpha} ; p_{\alpha \beta} ; \alpha \leqq \beta \in A\right\}$ where $A$ is closurefinite and each $P_{\alpha}$ is a compact metric $A N R$. Let $S$ be the collection of all countable subsets $s$ of $A$ such that for every $\alpha \in s$ there is a $\beta \in s, \alpha \leqq \beta$, with $p_{\alpha \beta}$ homotopic to a constant map. Since $P$ has trivial shape $A=\bigcup S$ and $S$ is directed by set containment. For each $s \in S$ let $\left\{P_{s} ; p_{\alpha}^{s} ; \alpha \in s\right\}$ be a presentation of $P_{s}$ as the limit of the inverse system $\left\{P_{\alpha} ; p_{\alpha \beta} ; \alpha \leqq \beta \in s\right\}$. $P_{s}$ is a compact metric space with trivial shape. Suppose $s, t \in S, s \subset t$, $\alpha, \beta \in s$ and $\alpha \leqq \beta$, then $p_{\alpha \beta} p_{\beta}^{t}=p_{\alpha}^{t}$. It follows that there is a continuous map $p_{s t}$ from $P_{t}$ to $P_{s}$ such that $p_{\alpha}^{s} p_{s t}=p_{\alpha}^{t}$ for all $\alpha \in s$, and this implies $\left\{P_{s} ; p_{s t} ; s, t \in S, s \subset t\right\}$ is an inverse system. Given $s \in S$, there is a continuous map $p_{s}$ from $P$ to $P_{s}$ such that $p_{\alpha}^{s} p_{s}=p_{\alpha}$ for all $\alpha \in S$. This implies $p_{s t} p_{t}=p_{s}$ for all $s, t \in S, s \subset t$. It is routine to show that $\left\{P ; p_{s} ; s \in S\right\}$ is a presentation of $P$ as the limit of the inverse system $\left\{P_{s} ; p_{s t} ; s, t \in S\right.$, $s \subset t\}$. The modifications of this argument for the case of pairs or pointed spaces are obvious.

1.4. THEOREM. If $P$, a compact Hausdorff space, has trivial shape, then $\left(P, P_{0}\right)$ has trivial shape for any $p_{0} \in P$.

Proof. In case $P$ is a metric space the result follows from 1.2, as each compact $A R$ is contractible to any point leaving that point fixed. If $P$ is not metrizable, the result follows from the first case, 1.3, and the continuity of the shape functor on the category of compact Hausdorff pairs. 
Given pointed spaces $\left(X, x_{0}\right)$ and $\left(P, p_{0}\right),\left(X, x_{0}\right) \wedge\left(P, p_{0}\right)$ will denote the quotient space obtained from $X \times P$ by identifying all points in the set $\left(\left\{x_{0}\right\} \times P\right) \cup\left(X \times\left\{p_{0}\right\}\right)$.

1.5. THEOREM. If $X, P$ are compact Hausdorff spaces, $P$ having trivial shape, $x_{0} \in X$ and $p_{0} \in P$, then $\left(X, x_{0}\right) \wedge\left(P, p_{0}\right)$ has trivial shape. If $X$ is arcwise connected so is $\left(X, x_{0}\right) \wedge\left(P, p_{0}\right)$.

Proof. Suppose $P$ is a metric space. By 1.2 there is a presentation $\left\{P ; p_{n} ; n=1,2, \cdots\right\}$ of $P$ as the limit of an inverse system of compact metric $A R$ 's $\left\{P_{n} ; p_{n, n+1} ; n=1,2, \cdots\right\}$. Each $P_{n}$ is contractible to $p_{n}\left(p_{0}\right)$ leaving $p_{n}\left(p_{0}\right)$ fixed. It follows that $\left(X, x_{0}\right) \wedge\left(P_{n}, p_{n}\left(p_{0}\right)\right)$ is contractible and therefore has trivial shape. By continuity of the shape functor $\left(X, x_{0}\right) \wedge$ $\left(P, p_{0}\right)$ has trivial shape. The nonmetric case follows from 1.3 and continuity of the shape functor. The last statement of the theorem is easily verified.

2. Homotopy groups. Suppose $\left\{\pi_{n}: n=1,2, \cdots\right\}$ is a collection of countable groups with $\pi_{n}$ abelian and admitting $\pi_{1}$ as a group of operators for $n \geqq 2$. It is shown in [8] that there is a locally finite connected polyhedron $X$ with $n$th homotopy group isomorphic to $\pi_{n}, n=1,2, \cdots$, in such a way that the action of the first group on the higher groups is preserved. The following lemma shows there is an arcwise connected compact metric space with this property.

2.1. Lemma. Suppose $X$ is a noncompact arcwise connected Hausdorff space such that $X=\bigcup\left\{K_{n}: n=1,2, \cdots\right\}$ where $K_{n}$ is compact and contained in the interior of $K_{n+1}$ for $n=1,2, \cdots$. Then $X$ can be embedded in an arcwise connected compact Hausdorff space whose homotopy groups are isomorphic to those of $X$ in such a way that the action of the first group on the higher groups is preserved. If $X$ is metric this space can be metric.

Proof. There is a continuous nonnegative real-valued function $f$ on $X$ such that $f(x) \geqq n$ for $x \in X-K_{n}$. Let $I$ be the interval $[-1,1]$ and $X_{0}$ the disjoint union of $X$ and $I$. Let open sets of $X$ be open in $X_{0}$. A basic open neighborhood of $y \in I$ in $X_{0}$ will consist of an open neighborhood $N$ of $y$ in $I$ and a set $\{x \in X-K: \sin (f(x)) \in N\}$ where $K$ is a compact subset of $X$. ( $X_{0}$ is a special case of the construction given in [5].) We will show that there are no arcs in $X_{0}$ connecting $X$ to $I$. Let $g=\sin \circ f$ on $X$ and $g(y)=y, y \in I$; then $g$ is a continuous map from $X_{0}$ to $I$. Let $f^{\prime}=f$ on $X$ and $f^{\prime}(y)=\infty$ for $y \in I$; then $f^{\prime}$ is a continuous map from $X_{0}$ to $[0, \infty]$. If $L$ is an arc in $X_{0},\left(f^{\prime} \times g\right)(L)$ is a path in $[0, \infty] \times I$ contained in $\{(t, \sin t): t \geqq 0\} \cup\{(\infty, y): y \in I\}$. Evidently $L$ is contained in $X$ or it is contained in $I$. We obtain the desired space by adding to $X_{0}$ an arc 
connecting a point in $X$ to a point in $I$. If $X$ is metric this space will also be metric.

In the next theorem we use the notation used in 1.5. $\wedge$ will denote the natural quotient map from $X \times P$ to $\left(X, x_{0}\right) \wedge\left(P, p_{0}\right)$.

2.2. THEOREM. An arcwise connected compact Hausdorff space $X$ can be embedded in an arcwise connected compact Hausdorff space $Y$ which has trivial shape yet homotopy groups isomorphic to those of $X$ in such a way that the action of the first group on the higher groups is preserved. If $X$ is metric, $Y$ can be metric.

Proof. Let $Q_{0}=\left\{\left(t, \sin t^{-1}\right): 0<t \leqq \pi^{-1}\right\}$ and $Q$ the closure in the plane of $Q_{0} . Q$ is the intersection of a decreasing sequence of compact $A R$ 's and so has trivial shape. If $q_{0} \in Q$ and $x_{0} \in X$ then by $1.5, Y=(X$, $\left.x_{0}\right) \wedge\left(Q, q_{0}\right)$ has trivial shape and is arcwise connected. If $X$ is metric so is $Y$. We will show that $X$ and $Y$ have isomorphic homotopy groups.

Given a topological space $Z$, let $Z^{\prime}$ denote the set $Z$ equipped with the weakest locally arcwise connected topology containing the original topology. A basis of open sets in this new topology is given by all arc components of all sets in any open basis of the original topology. (Details are contained in [1].) $Z$ and $Z^{\prime}$ have the same continuous maps from locally arcwise connected spaces [1, Theorem 3.2, p. 634]. We will show that $X^{\prime}$ is a strong deformation retract of $Y^{\prime}$ and then it follows that the homotopy groups of $Y$ are isomorphic to those of $X$ in such a way that the action of the first group on the higher groups is preserved.

Let $Q_{1}=Q-Q_{0}$ and take $q_{0} \in Q_{0}, q_{1} \in Q_{1}$. (We could take $q_{0} \in Q_{1}$ and $q_{1} \in Q_{0}$.) For $i=0,1$ let $f_{i}$ be a continuous map from $Q_{i} \times[0,1]$ to $Q_{i}$ such that $f_{i}(q, 0)=q, f_{i}(q, 1)=q_{i}$ and $f_{i}\left(q_{i}, t\right)=q_{i}$ for $q \in Q_{i}, t \in[0,1]$. For $i=0,1, q \in Q_{i}, x \in X$ and $t \in[0,1]$ define $f(\bigwedge(x, q), t)=\bigwedge\left(x, f_{i}(q, t)\right)$. Then $f$ is a strong deformation retraction of $Y^{\prime}$ onto the subspace $\left\{\bigwedge\left(x, q_{1}\right): x \in X\right\}$ which is homeomorphic to $X^{\prime}$. To see that $f$ is continuous let $x \in X-\left\{x_{0}\right\}$ and $q \in Q-\left\{q_{0}\right\}$. A typical basic open neighborhood of $\bigwedge(x, q)$ in $Y^{\prime}$ is a set of the form $\bigwedge\left(U^{\prime} \times V^{\prime}\right)$ where $U^{\prime}$ is the arc component of $x$ in an open set $U$ contained in $X-\left\{x_{0}\right\}$, and $V^{\prime}$ is the arc component of $q$ in an open set $V$ contained in $Q-\left\{q_{0}\right\}$. Such a set $\Lambda\left(U^{\prime} \times V^{\prime}\right)$ is contained in $\wedge\left(X \times Q_{0}\right)$ if $q \in Q_{0}, \wedge\left(X \times Q_{1}\right)$ if $q \in Q_{1}$. The continuity of $f_{0}$ and $f_{1}$ then imply the continuity of $f$ at $(\bigwedge(x, q), t)$ for $t \in[0,1]$. The continuity of $f$ at $\left(\wedge\left(x_{0}, q_{0}\right), t\right), t \in[0,1]$, is easily verified. This completes the proof.

From [8], 2.1 and 2.2 we have the following:

2.3. TheOREM. Suppose $\left\{\pi_{n}: n=1,2, \cdots\right\}$ is a collection of countable groups with $\pi_{n}$ abelian and admitting $\pi_{1}$ as a group of operators for $n \geqq 2$. 
There is an arcwise connected compact metric space with trivial shape and $n$th homotopy group isomorphic to $\pi_{n}$ for $n=1,2, \cdots$, in such a way that the action of the first group on the higher groups is preserved.

2.4. Remark. In [7] a definition is given for the shape groups $\pi_{n}\left(X, x_{0}\right), n=1,2, \cdots$, of $\left(X, x_{0}\right)$ a pointed compact Hausdorff space. The definition is shape invariant and if $X=\left\{x_{0}\right\}, \pi_{n}\left(X, x_{0}\right)$ is trivial for $n=1,2, \cdots$. It follows from 1.4 and 2.3 that an arcwise connected compact metric space can have trivial shape groups and quite arbitrary homotopy groups.

\section{REFERENCES}

1. A. M. Gleason and R. S. Palais, On a class of transformation groups, Amer. J. Math. 79 (1957), 631-648. MR 19, 663.

2. W. Holsztyński, An extension and axiomatic characterization of Borsuk's theory of shape, Fund. Math. 70 (1971), 157-168. MR 43 \#8080.

3. D. M. Hyman, On decreasing sequences of compact absolute retracts, Fund. Math. 64 (1969), 91-97. MR 40 \#6518.

4. J. Keesling, Shape theory and compact connected abelian topological groups (to appear).

5. P. A. Loeb, A minimal compactification for extending continuous functions, Proc. Amer. Math. Soc. 18 (1967), 282-283. MR 35 \#7301.

6. S. Mardesič and J. Segal, Shapes of compacta and ANR-systems, Fund. Math. 72 (1971), 41-59. MR 45 \#7686.

7. T. J. Sanders, Shape groups and products (preprint).

8. J. H. C. Whitehead, On the realizability of homotopy groups, Ann. of Math. (2) 50 (1949), 261-263. MR 10, 560.

Department of Mathematics, University of Florida, Gainesville, Florida 32601 